\title{
Standard cosmic ray energetics and light element production
}

\author{
B. D. Fields ${ }^{1}$, K. A. Olive ${ }^{2,3}$, M. Cassé , $^{4,5}$, and E. Vangioni-Flam ${ }^{5}$ \\ 1 Center for Theoretical Astrophysics, Department of Astronomy, University of Illinois, Urbana, IL 61801, USA \\ 2 TH Division, CERN, Geneva, Switzerland \\ 3 Theoretical Physics Institute, School of Physics and Astronomy, \\ University of Minnesota, Minneapolis, MN 55455 USA \\ 4 Service d'Astrophysique, CEA, Orme des Merisiers, 91191 Gif-sur-Yvette, France \\ 5 Institut d'Astrophysique, 98 bis Boulevard Arago, Paris 75014, France
}

Received 6 October 2000 / Accepted 15 February 2001

\begin{abstract}
The recent observations of an approximately linear relationship between both Be and B and iron in metal-poor stars has led to a reassessment of the origin of the light elements in the early Galaxy. In addition to standard secondary production of $\mathrm{BeB}$, it is necessary to introduce a production mechanism which is independent of the interstellar metallicity (primary), and in which freshly synthesized C, O and He are accelerated by supernova shock waves. Primary mechanisms are expected to be dominant at low metallicity. At metallicities higher than $[\mathrm{O} / \mathrm{H}] \gtrsim-1.75$, some existing data indicate that secondary production is dominant. In this paper, we focus on the secondary process, related to the standard Galactic cosmic rays, and we examine the cosmic ray energy requirements for both present and past epochs. We find the power input to maintain the present-day Galactic cosmic ray flux is about $1.510^{41} \mathrm{erg} / \mathrm{s}=510^{50} \mathrm{erg} /$ century; this estimate includes energy losses from both the escape of high-energy particle and ionization losses from low-energy particles. This implies that, if supernovae are the sites of cosmic ray acceleration, the fraction of explosion energy going to accelerated particles is about $\sim 30 \%$, a value which we obtain consistently both from considering the present cosmic ray flux and confinement and from the present ${ }^{9} \mathrm{Be}$ and ${ }^{6} \mathrm{Li}$ abundances. Using the abundances of ${ }^{9} \mathrm{Be}$ (and ${ }^{6} \mathrm{Li}$ ) in metal-poor halo stars, we extend the analysis to show the effect of the interstellar gas mass on the standard Galactic cosmic ray energetic constraints on models of $\mathrm{Li}, \mathrm{Be}$, and $\mathrm{B}$ evolution. The efficiency of the beryllium production per erg may be enhanced in the past by a factor of about 10; thus the energetic requirement by itself cannot be used to rule out a secondary origin of light elements. Only a clear and indisputable observational determination of the O-Fe relation in the halo will discriminate between the two processes.
\end{abstract}

Key words. ISM: cosmic rays - nuclear reactions, nucleosynthesis, abundances

\section{Introduction}

Energetic considerations have historically provided a fundamental constraint on and probe of the origin of cosmic rays. For example, energetic constraints have been used by many authors (see, e.g., the monograph by Brerezinskiu et al. 1990 and references therein) to point out that supernova (SN) explosions are a plausible cosmic ray power source, while other stars are not. An accounting of the energy budget has been particularly useful in the case of $\mathrm{Li}$, Be, and B (LiBeB) production. Ryter et al. (1970) used this type of argument to rule out in situ LiBeB production in young stars by flare particles (the "autogenetic" hypothesis), in favor of the present, "galactogenetic," hypothesis of LiBeB production by Galactic cosmic rays in the interstellar medium (Reeves et al. 1970).

Send offprint requests to: B. D. Fields, e-mail: bdfields@astro.uiuc.edu
Recently, an energetics argument has been invoked to discriminate between two possibilities, namely, a primary or a secondary origin of light elements in the halo (Ramaty et al. 1997). The appearance of a linear correlation between BeB and Fe in metal-poor stars led to the proposal of primary mechanisms (different from standard GCRN) for LiBeB production, in which $\mathrm{C}$, O nuclei ejected and accelerated by supernovae fragment on $\mathrm{H}$ and $\mathrm{He}$ at rest in the interstellar medium (e.g., Cassé et al. 1995; Ramaty et al. 1998; Vangioni-Flam et al. 1998; Higdon et al. 1998; Ramaty \& Lingenfelter 1999).

Given the approximately linear correlation between $[\mathrm{Be} / \mathrm{H}]$ and $[\mathrm{Fe} / \mathrm{H}]$, and a mean value of $\mathrm{Be} / \mathrm{Fe}$ of about $10^{-6}$, one can conclude that if $0.1 M_{\odot}$ of iron is produced per supernova, then about $210^{48}$ atoms of Be are produced per supernova. Standard secondary models of Be production yield an energy requirement of about $\sim 510^{3}$ ergs per Be atom at $[\mathrm{Fe} / \mathrm{H}]=-2.5$ or about 
$10^{52}$ ergs per supernova going into the production of Be. Of course this number is in excess of the total energy available in the supernova explosion. This is the basis of the energetics problem for secondary production and will be the main focus of this paper.

Note that the progenitor of $\mathrm{BeB}$ is not Fe but $\mathrm{O}$ (and $\mathrm{C})$. Thus the pertinent correlation is not between $\mathrm{BeB}$ and $\mathrm{Fe}$ but between $\mathrm{BeB}$ and $\mathrm{O}$. If $\mathrm{O} / \mathrm{Fe}$ is constant then the linearity between $\mathrm{BeB}$ and $\mathrm{Fe}$ translates directly into a linear relation between $\mathrm{BeB}$ and $\mathrm{O}$. In this case a primary mechanism is clearly required. The nature of the oxygen abundances in halo stars is currently controversial and depends on the lines used to derive the abundance; however, some recent observations (Israelian et al. 1998, 2001; Boesgaard et al. 1999b; Takeda et al. 2000; Mishenina et al. 2000) indicate that $[\mathrm{O} / \mathrm{H}]$ increases with decreasing $[\mathrm{Fe} / \mathrm{H}]$. In this case the linearity between $\mathrm{BeB}$ and Fe leads to the correlation $\log (\mathrm{Be} / \mathrm{H}) \sim 1.7[\mathrm{O} / \mathrm{H}]$, and should be explained by a combination of primary and secondary mechanisms (Fields \& Olive 1999a; Fields et al. 2000, hereafter FOVC). An unambiguous determination of the true $\mathrm{O} / \mathrm{Fe}$ trend in Pop II is crucial, and the results of the present study will only further underscore the need to resolve this issue.

It has been argued (Ramaty et al. 1997; Ramaty et al. 2000; Parizot 2000) that energetic arguments can be used to clarify the primary vs. secondary source for BeB. These authors have concluded that the secondary process in the early Galaxy meet with strong energetic difficulties. However, the energetics computation relies on many relatively uncertain factors and deserves a careful analysis (including the sensitivity to the injection energy spectrum to test the sensitivity of this criterion). Here, we will consider the energetics of standard Galactic cosmic rays in both the early and present Galaxy.

As we will see, a careful treatment of energetics allows us (1) to clarify and make precise the manner in which standard cosmic rays are to be included into chemical evolution, and then (2) to examine the viability of proposed cosmic ray origins. We begin by establishing (in Sect. 2) the present-day cosmic ray power budget, based on the observed properties of Galactic cosmic rays and of the Milky Way. By explicitly identifying supernova remnants as the power sources for the cosmic rays (in Sect. 3), we can express the present day energetics in terms of a cosmic ray acceleration efficiency per supernova (in Sect. 4). We find that this efficiency is subject to considerable uncertainties, due to uncertainties in the parameters describing cosmic rays, the Galaxy, and supernova blast energies. Nevertheless, the present efficiency must serve as a fiducial point against which we compare the past energetics as determined by Be (Sect. 5) and ${ }^{6} \mathrm{Li}$ (Sect. 6). In calculating the energetics of ${ }^{6} \mathrm{Li}$ and $\mathrm{Be}$, we note the importance of the larger gas mass in the early Galaxy relative to today, which implies a larger total number of atoms of all kinds in the interstellar medium. This in turn implies a larger number of target atoms (for a given metallicity) and thus a larger Be production rate, than one would estimate using the present day Galactic gas mass; the upshot is that one finds a higher Be production efficiency by a factor of $M_{\text {gas,init }} / M_{\text {gas,today }} \sim 10$ when one takes into account this gas-depletion effect (heretofore not considered in previous work). With this factor included, we find that the cosmic ray energetics implied by the Be data is in good agreement with present-day values. Indeed, we find that the energetics constraint is completely correlated with the basic constraint of agreement with Be-Fe data: models which fit the data also do not require more energy efficiency in the past. We discuss the implications of our results in Sect. 7.

Thus, the existence of an energetics problem will ultimately rest with new observations of Be at low metallicity. A dispersion of points above the secondary production predictions would indicate the need for a primary source of Be (perhaps occurring in spatially localized regions) to explain the high points. Barring that, the only true discriminant between primary and secondary production of Be at this time, will be a final and consistent determination of the $\mathrm{O} / \mathrm{Fe}$ ratio at low metallicity.

\section{Standard cosmic rays: Formalism and data}

The energy budget of Galactic cosmic rays is intimately connected with their acceleration, propagation, and losses in the Galaxy. Thus, we will follow the sources and sinks of the particles and the corresponding energy inputs and losses. The sources of Galactic cosmic rays are the events which accelerate them to their high energies. In this section, we will simply include the sources formally, saving comment on the physical acceleration mechanism (namely supernovae) until the next section. The sinks for standard cosmic rays are the losses due to escape from the Galaxy or energy deposition (ionization losses) in the ISM. These sources and sinks are described within a propagation model. The full, most realistic propagation equation is diffusive, following cosmic rays in space as well as time. However, for our purposes we will only need to follow the time history of the flux averaged over the Galaxy. Thus, we turn to the simplified propagation scheme of the "leaky box" model. Further refinements are in any case unwarranted by the approximations made in the chemical evolution portion of the analysis.

\subsection{Cosmic ray formalism}

To determine the propagated, interstellar particle spectrum we must first specify a (given) source spectrum. The source $q_{E}$ is defined so that the number of new particles (integrated over $4 \pi$ steradians) which are injected and accelerated to high energy in time interval $\mathrm{d} t$, volume $\mathrm{d} V$, and energy (per nucleon) range $(E, E+\mathrm{d} E)$ is $\mathrm{d} \mathcal{N}_{\text {inj }}=q_{E} \mathrm{~d} E \mathrm{~d} t \mathrm{~d} V$; thus $q_{E}$ is the number of particles accelerated per unit time in a unit volume and energy band. Similarly, the propagated particles have a spectrum $N_{E}$ defined by their number in a volume $\mathrm{d} V$ and energy band $\mathrm{d} E: \mathrm{d} \mathcal{N}_{\text {prop }}=N_{E} \mathrm{~d} E \mathrm{~d} V$. In other words, $N_{E}$ is the cosmic ray number density in energy band $(E, E+\mathrm{d} E)$. 
In the leaky box model, the source spectrum $q_{E}$ and the propagated spectrum $N_{E}$ are related by

$\frac{\partial N_{E}}{\partial t}=q_{E}-\frac{\partial}{\partial E}\left(b N_{E}\right)-\frac{1}{\tau} N_{E}$

where the $b=-\partial E / \partial t$ accounts for ionization energy losses and is defined so that $b>0$; for the numerical work below we will use the tabulations of Northcliffe \& Schilling (1970) and of Janni (1982). The effective timescale for inelastic nuclear losses and escape is $1 / \tau=1 / \tau_{\text {esc }}+1 / \tau_{\text {nuc }} \simeq$ $1 / \tau_{\text {esc }}$. Equation (1) holds for each cosmic ray species; of these, protons and $\alpha$-particles dominate the energy budget.

We will assume a steady state, $\partial N_{E} / \partial t=0$, which is valid as long as $q_{E}$ changes slowly with respect to the loss timescales; this is an excellent approximation for all but the very earliest moments in the Galaxy. In the steady state approximation, Eq. (1) is the basic relation used to solve for the propagated spectrum given the source spectrum and an escape time. It will also be useful to express $N_{E}$ in terms of the cosmic ray flux $\phi_{E}=N_{E} v$, which gives

$\frac{q_{E}}{\bar{\rho}}-\frac{\partial}{\partial E}\left(\omega \phi_{E}\right)-\frac{1}{\Lambda} \phi_{E}=0$

where $\omega=b / \bar{\rho} v$ and the cosmic ray mean path is $\Lambda=$ $\bar{\rho} v \tau_{\text {esc }} ; \bar{\rho}$ is the mean density encountered by the cosmic rays $^{1}$.

From these definitions, it follows that the cosmic ray injection power per unit volume is

$\langle E q\rangle=A \int_{0}^{\infty} \mathrm{d} E E q_{E}$

where the mass number $A$ accounts for the fact that $E$ is energy per nucleon. The total cosmic ray injection power is thus

$\dot{W}_{\mathrm{CR}}=\langle E q\rangle V=\frac{\langle E q\rangle}{\bar{\rho}} \bar{M}$

where $V$ is the volume in which the cosmic rays propagate. $\bar{M}=\bar{\rho} V$, the gas mass contained in $V$, and since most of the Galaxy's mass today is in the disk, $\bar{M} \approx M_{\mathrm{g}}$. In fact, $\dot{W}_{\mathrm{CR}}$ is the sum of these terms for each species in the cosmic rays.

\subsection{Cosmic ray data and energy requirements}

We can now estimate the energy input numerically for the present-day Galaxy, by evaluating each of the terms

\footnotetext{
1 The mean density can be determined today by comparing $\Lambda$ (as measured by the ratio of cosmic ray secondaries to primaries, e.g., B/C) and the mean lifetime of long-lived radioactive species in the cosmic rays ( $\operatorname{such}$ as ${ }^{10} \mathrm{Be}$ ). The result (e.g., Simpson \& Garcia-Munoz 1988; Lukasiak et al. 1994; Connell 1998 ) is that $\bar{\rho} \simeq 0.3 \rho_{\mathrm{g}}$, i.e., cosmic rays encounter a mean density about $1 / 3$ of that of the interstellar medium in the disk $\left(n_{\mathrm{g}} \simeq 1 \mathrm{~cm}^{-3}\right)$. This is usually interpreted to mean that the cosmic rays spend a significant part of their lives outside of the disk and in the halo, where the gas density is much lower.
}

on the right side of Eq. (4). Of these, the factors due to cosmic rays, $\langle E q\rangle / \bar{\rho}$, depends on the mean density and on the source spectrum we adopt. The source spectrum is itself determined from the observed spectrum, which is fairly well-measured at high energies by the local cosmic ray spectrum, but at low $(<100 \mathrm{MeV} / \mathrm{n})$ energies requires extrapolation and thus is model-dependent.

We adopt a source with a momentum (per nucleon) spectrum, $q_{p} \propto p^{-s} \mathrm{e}^{-E(p) / E_{\text {cut }}}$. This has the form of a power law in $p$, characteristic of shock acceleration, with a slope $2<s<3$. We choose a high-energy cutoff $E_{\text {cut }}=$ $10 \mathrm{TeV}$ to account for the maximum acceleration energy of a supernova (e.g., Ramaty et al. 2000). As long as $E_{\text {cut }} \gg$ $1 \mathrm{GeV}$, the integration of the spectrum and hence the value of $\dot{W}_{\mathrm{CR}}$ through Eqs. (3) and (4) is not very sensitive to the choice of $E_{\text {cut }}$. That is, most of the power in the integration comes at lower energies. To convert this to a kinetic energy spectrum we have $q_{E}=q_{p} \mathrm{~d} p / \mathrm{d} E=q_{p} / v$ and thus

$$
\begin{aligned}
q_{E} & =A v(E)^{-1} p(E)^{-s} \mathrm{e}^{-E / E_{\text {cut }}} \\
& =A(E+m)\left(E^{2}+2 m E\right)^{-(s+1) / 2} \mathrm{e}^{-E / E_{\text {cut }}}
\end{aligned}
$$

in units where $c=1$. This spectrum scales as $E^{-(s+1) / 2}$ in the non-relativistic regime, and as $E^{-s}$ in the relativistic regime. The constants $A$ and $s$ are set by noting that when energy losses can be neglected $(E \gg 100 \mathrm{MeV} / \mathrm{n})$, Eq. (2) gives $q_{E}=\phi_{E} \bar{\rho} / \Lambda$. This solution, along with measurements of $\phi_{E}$ and $\Lambda$, fixes $A$ and $s$.

To calculate the integrated spectrum, we must therefore first determine the parameters in Eq. (5), $A$ and $s$, from observations of $\phi_{E}$ and $\Lambda$. We first turn to the normalization A. Based on the compilation of Mori (1997), we take $\phi_{E_{1}}=2.8 \mathrm{~cm}^{-2} \mathrm{~s}^{-1} \mathrm{GeV}^{-1}$ at the (arbitrary) normalization energy $E_{1}=1 \mathrm{GeV}$. For the escape pathlength, we adopt the fit of Garcia-Muñoz et al. (1987), which has $\Lambda_{E_{1}}=9.2 \mathrm{~g} \mathrm{~cm}^{-2}$. This determines the normalization constant in Eq. (5) to be $A \simeq E_{1}^{s} \phi_{E_{1}} / \Lambda_{E_{1}}$.

The source spectral index $s$ is determined via the measured values of $\phi_{E}$ and $\Lambda(E)$; at high energies, each of these is found to behave as a power law, and thus $q_{E} \sim \phi_{E} / \Lambda(E)$ fixes $s$. Since $\Lambda(E)$ is itself determined by measured cosmic ray fluxes (e.g., the $\mathrm{B} / \mathrm{C}$ ratio), the source spectral index is best derived from a consistent analysis which yields both $s$ and $\Lambda(E)$. Several such analyses have been performed, giving values for $s$ ranging from 2.15 (Buckley et al. 1994) to 2.23 (Engelmann et al. 1990) to 2.3 (Webber 1998) to even 2.6 (Garcia-Muñoz et al. 1987). Here we wil adopt as a fiducial value the average of the three most recent analyses, $s=2.2$. One should bear in mind the allowed variation is at least \pm 0.1 .

Uncertainties are present in both the flux and the escape pathlength. The parameters for the flux are sensitive to the extrapolation from the observed, solar system intensity to the interstellar intensity. This extrapolation - "demodulation" - becomes important at $E \leq 1 \mathrm{GeV}$, and is model-dependent. Consequently, the combination of measurement errors and demodualtion procedure uncertainties leads to variation of about a factor of 5 (see, e.g., 
Mori 1997) in the quoted interstellar proton flux at $1 \mathrm{GeV}$, $\phi_{E_{1}}$, and uncertainties in the interstellar spectral index of order \pm 0.05 units. For example, the analysis of Webber (1998) gives $\phi_{E_{1}}=5 \mathrm{~cm}^{-2} \mathrm{~s}^{-1} \mathrm{GeV}^{-1}$ and uses a confinement which scales as $\beta R^{-0.6}$ (with $\beta=v / c$ and $R$ the rigidity) and has $\Lambda_{E_{1}}=10.7 \mathrm{~g} \mathrm{~cm}^{-2}$; for $s=2.1$, this gives an $A$ about $25 \%$ larger than our adopted value.

Both the non-relativistic and relativistic limits show that the input power integral $\langle E q\rangle / \bar{\rho}=\int \mathrm{d} E E q_{E}$ has its maximum contribution in the $E_{\max } \sim m$ regime, and taking the dominant contribution from the relativistic limit, we find

$$
\begin{aligned}
\langle E q\rangle / \bar{\rho} & \simeq Y \frac{\left(E_{1} / m\right)^{s-2}}{s-2} \frac{E_{1}^{2} \phi_{E_{1}}}{\Lambda_{E_{1}}} \\
& \simeq 5 Y \frac{E_{1}^{2} \phi_{E_{1}}}{\Lambda_{E_{1}}}=2.1 \mathrm{GeV} \mathrm{g}^{-1} \mathrm{~s}^{-1}
\end{aligned}
$$

where the latter expressions use $s=2.2$. The factor

$Y=\sum A_{i} y_{i}^{\mathrm{CR}} \approx 1+4(\mathrm{He} / \mathrm{p})_{\mathrm{CR}} \simeq 1.4$

includes the additional contribution due to $\mathrm{He}$ (with $\mathrm{He} / \mathrm{H} \sim 0.1$ ), with the mass number $A_{i}$ converting from energy per nucleon to energy, and where $y_{i}^{\mathrm{CR}}=\phi_{E}^{i} / \phi_{E}^{p}$ is the abundance of species $i$ in the cosmic rays. We see that the input power scales as $\phi_{E_{1}} / \Lambda_{E_{1}}$, as it should, and the the input power density is fixed by local measurements of the cosmic ray flux and escape pathlength. We can thus analytically estimate $\langle E q\rangle / \bar{n}=m_{\mathrm{p}}\langle E q\rangle / \bar{\rho}=$

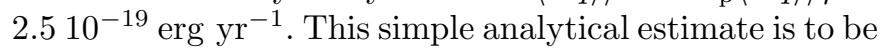
compared with the results using a full numerical integration of the source spectrum for both protons and He, which

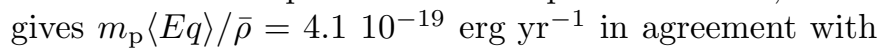
our rough analytical estimate ${ }^{2}$. If $\bar{M} \simeq M_{\mathrm{g}} \sim 10^{10} M_{\odot}$, then this gives

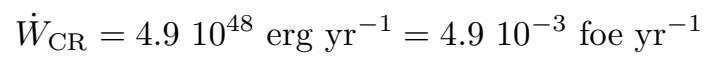

or $1.510^{41} \mathrm{erg} / \mathrm{s}$. This is similar to other estimates; e.g., Drury et al. (1989), find $\dot{W}_{\mathrm{CR}}=1.110^{41} \mathrm{erg} / \mathrm{s}$, using a $p^{-2.2}$ spectrum but somewhat different gas mass and confinement. We note that, for a $p^{-2.2}$ spectrum, about $20 \%$ of $\dot{W}_{\mathrm{CR}}$ comes from source energies below $1 \mathrm{GeV}$; this fraction rapidly increases for a steeper spectrum, reaching $45 \%$ for $p^{-2.5}$. Many of these lower-energy particles are stopped via ionization losses to the ISM rather than by loss from the Galaxy. The contributions to particles such as these are not counted in many estimates of $\dot{W}_{\mathrm{CR}}$ which only include the contributions due to escape losses, but are important and are included in our results.

The result for $\dot{W}_{\mathrm{CR}}$ has significant uncertainties tracing back to the input parameters. As noted above, the uncertainties due to cosmic ray absolute flux calibrations,

\footnotetext{
2 The main difference comes from the differences in the adopted spectrum (Eq. (5)): and its relativistic approximation as $A E^{-\gamma}$ used in the analytic estimate. When the two are both normalized to $\phi_{E_{1}}$ at $E_{1}$, the power law approximation is lower for all $E>E_{1}$, accounting for most of the discrepancy.
}

spectral index determinations, and demodulation corrections, which easily allow for about a factor of 4 variation in $A$ (Mori 1997). As a result, $\langle E q\rangle / \bar{n}$ can vary by a factor of 3 within the current uncertainties. As an illustration of the possible variations, and to allow comparison to previous work, we turn to the spectra of Ramaty et al. (2000) and Parizot (2000). The Ramaty spectrum has $s=2.5$, $E_{\text {cut }}=10 \mathrm{GeV} / \mathrm{amu}$, and $\Lambda(E)=10 \mathrm{~g} \mathrm{~cm}^{-2}$. To compare with our results, we choose the same flux normalization as our fiducial estimate, $\phi_{E_{1}}=2.8 \mathrm{~cm}^{-2} \mathrm{~s}^{-1} \mathrm{GeV}^{-1}$. In this case, we find $\langle E q\rangle / \bar{n}=1.710^{-19} \mathrm{erg} \mathrm{yr}^{-1}$. This is about $40 \%$ of our result. The Parizot spectrum is $q_{E} \propto E^{-1} \mathrm{e}^{-E / E_{\text {cut }}}$, with $E_{\text {cut }}$ varying from $10 \mathrm{MeV}$ to $500 \mathrm{MeV}$. This is meant to describe acceleration within superbubble shocks. Again, for comparison we normalize to $\phi_{E_{1}}=2.8 \mathrm{~cm}^{-2} \mathrm{~s}^{-1} \mathrm{GeV}^{-1}$. For $E_{\text {cut }}=500 \mathrm{MeV} / \mathrm{amu}$,

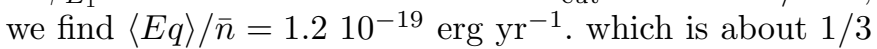
of our result. On the other hand, the $E_{\text {cut }}=10 \mathrm{MeV} / \mathrm{amu}$ is a low-energy spectrum which does not resemble the observed GCR flux and should not be used in this context. In particular, fixing the normalization to the observed flux at our $E_{1}=1 \mathrm{GeV}$ implies that the flux at, say, $E=10 \mathrm{MeV}$ is higher by a factor of $\exp \left(E_{1} / E_{\text {cut }}\right)=e^{100}$, with a similarly large increase in $\langle E q\rangle / \bar{n}$.

Finally, there is significant uncertainty in the present Galactic gas mass. We have used $M_{\text {gas }}=10^{10} M_{\odot}$. Smaller estimates exist: Henderson et al. (1982) infer a neutral atomic hydrogen content of $M_{\mathrm{HI}}=4.810^{9} M_{\odot}$; including a helium component with mass fraction $\sim 0.25$ gives $6.410^{9} M_{\odot}$. The total gas mass should include the molecular and ionized components, which each are likely to be at least $\sim 25 \%$ of the neutral atomic component and thus lead to the estimate we use. The neutral atomic mass is inferred from $21-\mathrm{cm}$ radio observations, which are themselves quite accurate, but in terms of column densities. To arrive at a mass requires assumptions regarding the distribution of gas in the Galaxy, which leads to uncertainties of at least a factor of 2 . Including the gas mass uncertainty with those in the cosmic ray inputs, the estimate of $\dot{W}_{\mathrm{CR}}$ can easily vary by a factor of 5 .

To summarize: we have quantified the power requirement $\dot{W}_{\mathrm{CR}}$, and computed the present-day value give the flux at Earth. However we have so far made no statement about the physical nature of the energy source. We now turn to this issue.

\section{Supernovae as sources of cosmic rays}

We now invoke supernovae as the source - the acceleration engine - of cosmic rays. This connection has both observational and theoretical support. Observations of supernova remnants confirm that these environments are excellent particle accelerators. Radio synchrotron emission has long been observed for supernova remnants and establishes that nonthermal, relativistic electrons are accelerated in these environments. The detection of X-ray synchrotron radiation (Koyama et al. 1995), and $\mathrm{TeV} \gamma$-rays (Tanimori et al. 1998) both confirm that the electrons are 
accelerated up to $\sim 100 \mathrm{TeV}$. In additional, recent $\gamma$-ray observations provide the first direct evidence of nucleon acceleration to $E>300 \mathrm{MeV} / \mathrm{n}$ in supernova remnants (Combi et al. 1998). These observations are in broad agreement with the predictions of theoretical models of particle acceleration in supernova shocks, and thus a coherent picture is emerging in which it seems that supernovae are capable of accelerating particles at least to $\sim 100 \mathrm{TeV}$.

The net Galactic cosmic ray injection power due to supernovae can be written as

$\dot{W}_{\mathrm{SN}}=\eta_{\mathrm{CR}} E_{\mathrm{SN}} \dot{\mathcal{N}}_{\mathrm{SN}}$

where $E_{\mathrm{SN}}$ is the average mechanical energy output of a supernova, $\dot{\mathcal{N}}_{\mathrm{SN}}$ is the Galactic supernova rate, and $\eta_{\mathrm{CR}}=E_{\mathrm{CR}} / E_{\mathrm{SN}}$ is the cosmic ray acceleration efficiency - the fraction a supernova explosion's total mechanical energy which goes into cosmic rays.

The appearance of the supernova rate in Eq. (9) will allows us to insert the cosmic ray energy budget - and thus the cosmic ray sources and flux - within the chemical evolution formalism in a straightforward way. In chemical evolution, the supernova rate is related in a simple way to the fundamental input, the global star formation rate $\psi$. Specifically,

$$
\begin{aligned}
\dot{\mathcal{N}}_{\mathrm{SN}} & =\int_{\mathrm{SN}} \mathrm{d} m \xi(m) \psi\left(t-\tau_{m}\right) \\
& \simeq \psi(t) \int_{\mathrm{SN}} \mathrm{d} m \xi(m)=X_{\mathrm{SN}} \psi /\langle m\rangle_{\mathrm{SN}}
\end{aligned}
$$

where $\xi(m)$ is the initial mass function and $\tau_{m}$ is the age of a star of mass $m$, and "SN" denotes the mass range of supernova progenitors, $m \gtrsim 8-10 M_{\odot}$. The approximation in the last two expressions holds if $t \gg \tau_{m_{\mathrm{SN}}} \sim$ $10 \mathrm{Myr}$, which is an excellent approximation for all but the very earliest moments of Galactic history. The supernova mass fraction is $X_{\mathrm{SN}}=\int_{\mathrm{SN}} \mathrm{d} m m \xi(m) / \int \mathrm{d} m m \xi(m)$ and the mean supernova progenitor mass is $\langle m\rangle_{\mathrm{SN}}=$ $\int_{\mathrm{SN}} \mathrm{d} m m \xi(m) / \int_{\mathrm{SN}} \mathrm{d} m \xi(m)$. For a Salpeter IMF, $X_{\mathrm{SN}}=0.14$, and $\langle m\rangle_{\mathrm{SN}}=19 M_{\odot}$, and thus the supernova and star formation rates are connected by $\dot{\mathcal{N}}_{\mathrm{SN}}=$ $7.410^{-3} \psi / M_{\odot}$. Thus, if the Galactic supernova rate is about $(3 \pm 2) 10^{-2} / \mathrm{yr}$, as in a recent estimate (Dragicevich et al. 1999), then one would infer a star formation rate $\psi=4 M_{\odot} / \mathrm{yr}$, in general agreement with other estimates (e.g., Timmes et al. 1997 and Refs. therein; Scalo 1986 and Refs. therein).

Thus, we have the scaling $\dot{W}_{\mathrm{SN}} \propto \psi$, with the explicit relation

$\dot{W}_{\mathrm{SN}}=\eta_{\mathrm{CR}} E_{\mathrm{SN}} X_{\mathrm{SN}} \psi /\langle m\rangle_{\mathrm{SN}}$.

Inserting the Galactic supernova rate into (9) or (11), we have

$\dot{W}_{\mathrm{SN}}=310^{-2} \eta_{\mathrm{CR}}$ foe $\mathrm{yr}^{-1}$

where we use $E_{\mathrm{SN}}=10^{51} \mathrm{erg} \equiv 1$ foe, and $\dot{\mathcal{N}}_{\mathrm{SN}}=$ $3(100 \mathrm{yr})^{-1}$; these are good fiducial numbers but are each subject to uncertainties of at least a factor of 2 .

\section{Cosmic ray efficiency in the present epoch}

Now we can unambiguously link the cosmic ray and chemical evolution formalisms. The connection is made by asserting that supernovae are the cosmic ray accelerators. To implement this statement we join Eqs. (4) and (11):

$\dot{W}_{\mathrm{CR}}=\dot{W}_{\mathrm{SN}}$.

This equation has important implications, which we now explore. We first turn to the specific numerical implementation of this statement and estimate the present-day cosmic ray acceleration efficiency. We then elaborate on how this statement clarifies the formalism used to date in treatments of cosmic rays in chemical evolution.

\subsection{The present-day cosmic ray efficiency}

Equations (8), (12), and (13) together imply that the present-day cosmic ray efficiency is

$\eta_{\mathrm{CR}}=0.16$

i.e., $16 \%$, if the supernova mechanical energy is $E_{\mathrm{SN}}=$ 1 foe. We caution that this result is not known to high accuracy, due to the uncertainties in the inputs, notably the supernova energy, rate, and present gas mass (which also plague previous estimates). If one allows the input parameters to vary within plausible ranges, $\eta_{\mathrm{CR}}$ can easily span more than order of magnitude, from $<10 \%$ to $100 \%$ or more. (Of course as $\eta_{\mathrm{CR}}$ approaches 1, we have the unphysical result that from the point of view of Eq. (13), cosmic rays require more than the available mechanical energy in the supernova.)

However, it is encouraging that our fiducial result is reasonable and suggestive when one recalls that the cosmic ray energy density in the ISM is in rough equipartition with the thermal and magnetic energy. In other words, it is well known that the propagated particles reach an energy balance with their environment. Here, we see that even in the acceleration process there is a rough equipartition of energy between the accelerated particles and the other components. This empirical figure is in line with theoretical estimates of particle acceleration efficiencies in supernova remnants. For example, Berezhko \& Volk (1997) recently conclude that the acceleration efficiency could reach $50 \%$. It is also worth noting that this result is directly tied to the local cosmic ray flux. Consequently, a similar result would be obtained in all models which fit the observed properties of the comic ray flux and propagation. That is, this result is model-independent as an order of magnitude estimate, but the precise value depends on the details of the inputs used.

\subsection{Scaling laws for cosmic ray flux}

Equation (13) implies that

$q=\eta_{\mathrm{CR}} \frac{E_{\mathrm{SN}}}{\langle E\rangle_{\mathrm{inj}}} X_{\mathrm{SN}} \frac{\psi}{\langle m\rangle_{\mathrm{SN}} V}$ 
where the mean injection energy $\langle E\rangle_{\mathrm{inj}}=A \int \mathrm{d} E E q_{E} /$ $\int \mathrm{d} E q_{E}$ depends on the shape of the source spectrum. Further, since the integrated cosmic ray flux is proportional to the integrated source strength, $\phi \propto q$, we have

$\phi \propto \frac{\psi}{V}$.

Thus, for a fixed volume, a higher supernova rate means a higher star formation rate and more cosmic ray acceleration; this in turn means higher flux (for a fixed confinement). To understand the appearance of $V$ in Eq. (16), imagine dividing the leaky box volume into two identical subregions; each of these will have both a star formation rate and a volume that is half of the larger region (recall that $\psi$ represents the total star formation rate in $M_{\odot} /$ unit time and is in effect proportional to $V$ ). Subdividing the volume in this way should not affect the cosmic ray flux, and Eq. (16) guarantees that it does not. Finally, if the leaky box volume is fixed-a good approximation today, though it may not be for early Galaxy-then we have $\phi \propto \psi$, as is usually assumed in the literature.

It is important to note that in the early Galaxy, which gave rise to Pop II stars, conditions were likely different from today. These differences can affect not only cosmic ray injection, but also cosmic ray propagation. To see this, note that the cosmic ray flux is related to the injection and confinement roughly by $\phi \sim \Lambda q / \bar{\rho}$, which generalizes Eq. (16) to

$\phi \propto \Lambda \psi / \bar{\rho} V$.

Thus, the flux strength depends not only on the supernova rate and the volume $V$ of the confinement region, but also on the mean escape length $\Lambda / \bar{\rho}$. This ratio (as well as the volume) could have been different in the early Galaxy. For example, it is very likely that the early Galaxy as a whole had a larger volume (before it settled to a disk), it seems likely that the cosmic ray confinement volume was also larger. The cosmic ray escape time would likely have been different as well. The escape losses depend on the interplay between the ISM density and the Galactic magnetic field, and as it is unclear how these would have varied, and consequently difficult to see whether $\Lambda / \bar{\rho}$ would have been larger or smaller. Clearly it is difficult to ascribe a realistic uncertainty on this quantity. Although such a study would be very useful, and would modify the simple scaling of cosmic ray flux with the supernova rate, it is clearly beyond the scope of this paper and we will retain the $\phi \propto \psi$ scaling. Another uncertainty concerns the increased gas mass and thus enhanced total number of ISM atoms in the halo phase (see below, Sect. 5). It could well be that the stars in which $\mathrm{LiBeB}$ is observed have been formed in the thick Galactic disk.

Indeed this scaling of $\phi \propto \psi$ has been made by most previous work early Galactic cosmic ray nucleosynthesis. Its common use is evidence for its intuitive appeal. Note that here, we have derived this scaling. It is demanded by considerations of energetics together with the explicit assumption of supernovae as the acceleration engines of

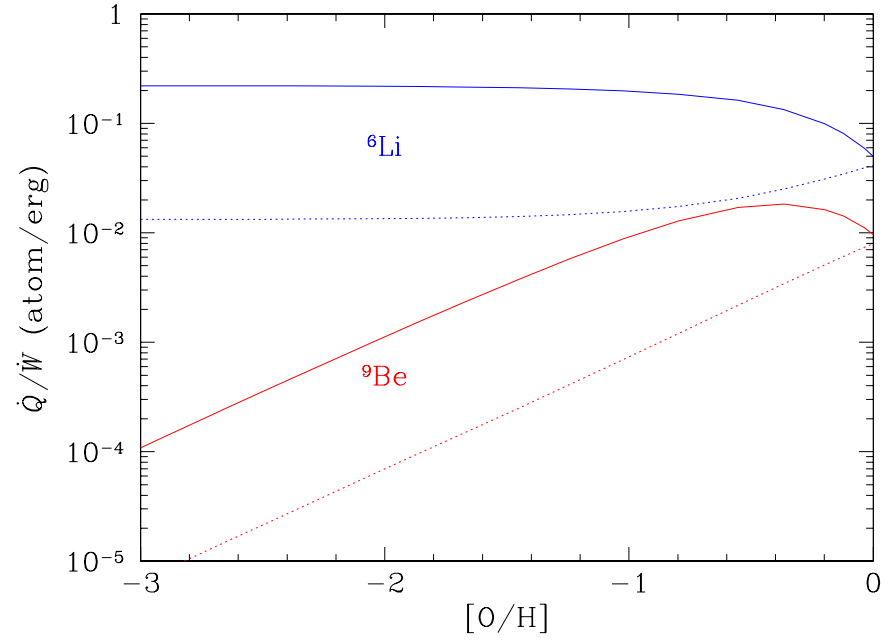

Fig. 1. A plot of $\dot{Q}_{\mathrm{Be}} / \dot{W}_{\mathrm{CR}}$ for our model. Dotted lines: constant $N_{\mathrm{H}} ;$ full lines: evolving $N_{\mathrm{H}}$

Galactic cosmic rays. Thus we see again that considerations of energetics help to clarify issues of cosmic ray origin.

\section{Beryllium and cosmic ray efficiency}

So far, we have concentrated on cosmic ray energetics today. Now we turn to cosmic ray energetics in the past. Our approach follows the basic argument of Ramaty et al. (1997, 2000), which we extend by including aspects of the early Galactic environment that were not heretofore emphasized. (Also, our adopted source spectrum is somewhat different, which can be important in the comparison of numerical results.)

The instantaneous cosmic ray input energy per supernova is

$\epsilon \equiv \frac{\Delta W_{\mathrm{CR}}}{\Delta \mathcal{N}_{\mathrm{SN}}}=\frac{\dot{W}_{\mathrm{CR}} \Delta t}{\dot{\mathcal{N}}_{\mathrm{SN}} \Delta t}=\frac{\dot{W}_{\mathrm{CR}}}{\dot{\mathcal{N}}_{\mathrm{SN}}}$

for each epoch $t$, where $W_{\mathrm{CR}}$ and $\mathcal{N}_{\mathrm{SN}}$ are the aggregate injection energy and supernova number over the Galactic history. From (18), we can relate $\epsilon$ to the cosmic ray efficiency: $\epsilon=\eta_{\mathrm{CR}} E_{\mathrm{SN}}$. To see this relation more clearly, consider models where supernovae power cosmic ray acceleration. Then the total injection power is fixed by the scaling $\dot{W}_{\mathrm{CR}} \propto q V \propto \epsilon \psi$, normalized to the present value as calculated above. Since $\dot{\mathcal{N}}_{\mathrm{SN}} \propto \psi$ as well, Eq. (18) follows directly. We will assume that $\epsilon$ is constant in time, and use the Be-O data to check this assumption.

The observed Be/O ratio is connected to the energetics via $\dot{W}_{\mathrm{CR}} / \dot{N}_{\mathrm{Be}}$, the ratio of the Galactic cosmic-ray power input to the Be production rate $\dot{N}_{\mathrm{Be}}$. As is well-described in the literature, the total Galactic Be mass changes according to the usual chemical evolution expression

$\mathrm{d} M_{\mathrm{Be}} / \mathrm{d} t=m_{\mathrm{Be}} \dot{Q}_{\mathrm{Be}}-X_{\mathrm{Be}} E \simeq m_{\mathrm{Be}} \dot{N}_{\mathrm{Be}}$

Here, $\dot{Q}_{\mathrm{Be}}$ is the Be source rate, i.e., the total number of Be atoms produced by cosmic rays per second. 
The mass fraction of $\mathrm{Be}$ is $X_{\mathrm{Be}}$, and the ejection function $E=\int \mathrm{d} m \psi\left(t-\tau_{m}\right) \phi(m)$ quantifies the rate at which gas mass is expelled from dying stars. The last approximate equality is valid at early times (metallicities less than solar) when Be astration can be neglected. Thus, at early times, $\dot{W}_{\mathrm{CR}} / \dot{N}(\mathrm{Be}) \simeq \dot{W}_{\mathrm{CR}} / \dot{Q}_{\mathrm{Be}}$. This ratio is just the inverse of the $Q / W$ ratio of Ramaty et al. (2000), up to the differences in the observed spectra. The $\dot{N}(\mathrm{Be}) / \dot{W}_{\mathrm{CR}}$ ratio depends on the $\mathrm{CNO}$ abundances in the ISM since $\dot{N}(\mathrm{Be}) \propto \mathrm{O} / \mathrm{H}$.

At early times, therefore, the Be production rate is just given by the source rate

$\mathrm{d} N_{\mathrm{Be}} / \mathrm{d} t=\dot{Q}_{\mathrm{Be}}=\sum_{i j} N_{i}\left\langle\sigma_{i j} \phi_{j}\right\rangle$

where the sum runs over targets $i$ and CR species $j$, and $N_{i}$ is the number of Galactic gas atoms in the form of $i$ : $N_{i}=n_{i} V$. It is important to note that the $n_{i}$ refer to the gas density of in the Galaxy proper, i.e., in regions whose material will be incorporated into star forming regions and ultimately in Pop II stars; this density is not identical to the mean density $\bar{n}$ seen by cosmic rays on their excursions in and out of the gas-rich parts of the Galaxy.

In standard cosmic ray nucleosynthesis, the main target is $\mathrm{O}$, and the main projectiles are protons. Furthermore, the cosmic ray species are assumed to occur in with the same spectral shapes, only different relative abundances, so that $\phi_{E}^{i}=y_{i}^{\mathrm{CR}} \phi_{E}^{p}$. Thus we can rewrite

$\mathrm{d} N_{\mathrm{Be}} / \mathrm{d} t=\dot{Q}_{\mathrm{Be}}=\frac{\mathrm{O}}{\mathrm{H}} N_{\mathrm{H}} \Phi_{\mathrm{p}} \bar{\sigma}$

where $\Phi_{\mathrm{p}}=\int \mathrm{d} E \phi_{E}$ is the total integrated flux in protons, and

$\bar{\sigma}=\sum_{i j} A_{i} / \mathrm{O} y_{i}^{\mathrm{CR}}\left\langle\sigma_{i j} \phi\right\rangle / \Phi_{\mathrm{p}}$

is an abundance - and spectrum - weighted cross section, with $A_{i} \in \mathrm{CNO}$.

Thus we have

$$
\mathrm{d} N_{\mathrm{Be}} / \mathrm{d} t \sim(\mathrm{O} / \mathrm{H}) N_{\mathrm{H}} \sigma \phi \propto(\mathrm{O} / \mathrm{H}) N_{\mathrm{H}} \sigma \psi
$$

that is, the product of the number of targets (i.e., $N_{\mathrm{O}}=$ $\left.(\mathrm{O} / \mathrm{H}) N_{\mathrm{H}}\right)$, the appropriate spallation cross sections, and the cosmic ray flux. The key feature here is the appearance of $N_{\mathrm{H}}$, the total number of $\mathrm{H}$-atoms in the Galactic gas, which encodes the fact that what matters is the absolute number of targets and not just their relative abundance. Note that $N_{\mathrm{H}}=M_{\text {gas }}(\mathrm{H}) / m_{\mathrm{p}}=X_{\mathrm{H}} M_{\text {gas }} / m_{\mathrm{p}}$, and since $X_{\mathrm{H}}$ varies by only a few percent over the history of the Galaxy, then $N_{\mathrm{H}} \propto M_{\text {gas }}$. Thus we have

$\dot{Q}_{\mathrm{Be}} / \dot{W} \sim \frac{(\mathrm{O} / \mathrm{H}) N_{\mathrm{H}} \bar{\sigma} \phi}{\langle E q\rangle \bar{\rho} V} \sim \frac{(\mathrm{O} / \mathrm{H}) N_{\mathrm{H}} \bar{\sigma}}{\langle E\rangle_{\text {inj }} \bar{\rho} V / \Lambda} \sim Z M_{\text {gas }}$

where the second expression uses the mean injection energy defined after Eq. (15) and the approximation $q \sim$ $\phi / \Lambda$. The last expression uses the chemical evolution language of metallicity $Z \propto \mathrm{O} / \mathrm{H}$ and gas mass, and makes the usual assumption that $\Lambda$ (and thus $\bar{\rho}$ ) is constant.
The appearance of $N_{\mathrm{H}}$ (or equivalently, gas mass) in $\dot{Q} / \dot{W}$ has not been heretofore emphasized, but plays a key role in the energetics, since the gas content (absolute number of atoms) of the Galaxy decreases with time, while the metallicity increases. One must include both effects to fully evaluate the energetics. Figure 1 illustrates the effect of the $M_{\text {gas }}$ factor; without it (dashed curves) one simply has $\dot{N}_{\mathrm{Be}} / \dot{W} \propto Z$, and the plot is a straight line. However, with it, the solid curves show that there is a peak where the product of decreasing gas mass and increasing metallicity is maximized ${ }^{3}$. Moreover, at low metallicity, the curve has the same linear shape, but is increased by the factor $M_{\text {gas,init }} / M_{\text {gas,now }} \sim 10$. This large factor plays a key role in the energetics.

We can thus make a rough analysis of Be energetics in the early Galaxy. This argument was proposed and developed by Ramaty et al. $(1997,2000)$; the detailed treatment here closely follows and extends that of Fields \& Olive (1999a) and FOVC. The basic idea is that the abundances of Be and $\mathrm{O}$ at any epoch are a record of the total cosmic ray and supernova energy inputs, respectively, for that epoch. Thus an analysis of the evolution of the Be/O ratio can shed light on the evolution of the cosmic ray energy budget.

The cosmic ray input energy per supernova is thus calculable given (1) the "energy per atom" ratio, (2) the observed Be/O ratio, and (3) the oxygen output per supernova. Specifically, the following expression relates these quantities:

$\frac{\dot{W}_{\mathrm{CR}}}{\dot{\mathcal{N}}_{\mathrm{SN}}}=\left[\frac{\dot{W}_{\mathrm{CR}}}{\dot{N}(\mathrm{Be})}\right]_{\mathrm{th}}\left[\frac{\dot{N}(\mathrm{Be})}{\dot{N}(\mathrm{O})}\right]_{\mathrm{obs}}\left[\frac{\dot{N}(\mathrm{O})}{\dot{\mathcal{N}}_{\mathrm{SN}}}\right]_{\mathrm{th}}$.

Note that, strictly speaking, the $\dot{N}(\mathrm{Be}) / \dot{N}(\mathrm{O})$ term is in fact the ratio of $\mathrm{Be}$ and $\mathrm{O}$ production rates in the theory. However, as long as astration effects are unimportant and the $\mathrm{Be}$ and $\mathrm{O}$ data are related by a power law so $\mathrm{Be} \propto$ $\mathrm{O}^{\omega_{\mathrm{BeO}}}$, then

$\dot{N}(\mathrm{Be}) / \dot{N}(\mathrm{O})=\omega_{\mathrm{BeO}}(\mathrm{Be} / \mathrm{O})_{\mathrm{obs}}$

In this way, one can use the observed Be trends versus $\mathrm{O}$ (or $\mathrm{Fe}$ ) to constrain the cosmic ray energy input per supernova over all epochs for which abundances are available. At low-metallicities, the $\mathrm{Be}-\mathrm{O}$ trend has $\omega_{\mathrm{BeO}} \simeq 1.7 \pm 0.2$ (using the ultraviolet $\mathrm{OH}$ lines; see below).

Let us apply this analysis to Be-O data. Figure 2 plots $\mathrm{Be} / \mathrm{O}$ for halo stars. Both the $\mathrm{Be}$ and the $\mathrm{O}$ data points are adjusted to the consistent, "Balmer" set of stellar parameters described in $\mathrm{FOVC}^{4}$. $\mathrm{FOVC}$ showed that the

\footnotetext{
${ }^{3}$ In the instantaneous recycling approximation, we have $M_{\text {gas }}=M_{\text {gas,init }} \mathrm{e}^{-Z / y}$, with $y$ the yield. Thus $Q / W \sim Z \mathrm{e}^{-Z / y}$, which is maximized at $Z=y$.

${ }^{4}$ We have only included data for stars which have both Be and $\mathrm{O}$ data available. Recently, Be data has been published (Primas et al. 2000; Primas et al. 2000) for three new stars with $[\mathrm{Fe} / \mathrm{H}] \lesssim-3$. With the addition of $\mathrm{O}$ abundances, these stars can clarify the crucial low- $[\mathrm{O} / \mathrm{H}]$ region of the data.
} 


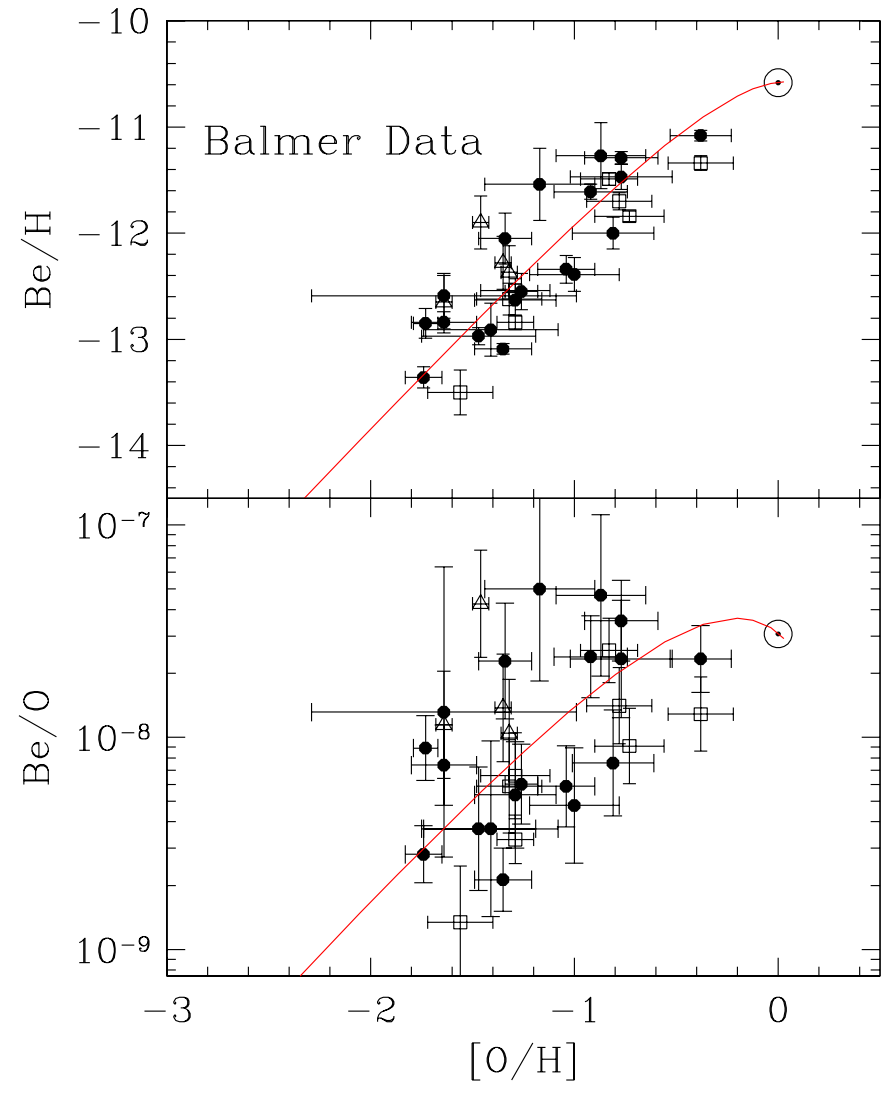

Fig. 2. Be and O data, derived from a consistent set of stellar atmospheres using the "Balmer" method described in Fields et al. (2000) (see discussion in text). The curve is for a model in which Be is produced by standard Galactic cosmic rays. Be data from Rebolo et al. (1988, 1993); Ryan et al. (1990, 1992); Gilmore et al. (1992); Boesgaard \& King (1993); Primas (1995); Hobbs \& Thorburn (1996); Molaro et al. (1997); and Boesgaard et al. (1999b)

basic Be/O-O trend seen in Fig. 2 is robust to changes in the data sets and atmospheric parameters one adopts, though the detailed slopes do vary slightly. As we have noted above, and discussed in detail in FOVC, the question of halo star $\mathrm{O}$ abundances-and hence $\mathrm{O} / \mathrm{Fe}$ ratios-is currently controversial. The $\mathrm{O}$ abundances in Fig. 2 are derived using the $\mathrm{OH}$ lines around $3100 \AA$. Oxygen abundances using these lines show (Israelian et al. 1998, 2001; Boesgaard et al. 1999b) an increasing O/Fe trend towards low $[\mathrm{Fe} / \mathrm{H}]$. This result has been supported by observations (Takeda et al. 2000; Mishenina et al. 2000) of the permitted O I lines at $7771 \AA$.

On the other hand, a preliminary analysis (Asplund 2000) with a 3-D atmospheric model suggests that the abundances using the $\mathrm{OH}$ data may have to be corrected downwards. Non-LTE corrections to these abundances are likely to be important, but the size of the correction is uncertain, as indicated by differences between the $\mathrm{O} / \mathrm{Fe}$ trends found in the recent studies by King (2000), Israelian et al. (2001), Carretta et al. (2000), and Gratton et al. (2000). Finally, the $\mathrm{O}$ abundances derived from the $\mathrm{OH}$ and permitted O I lines may (e.g., Fulbright \& Kraft 1999) or may not (e.g., Israelian et al. 2001) differ systematically. Clearly, a resolution of the true $\mathrm{O}$ abundance indicator is urgently needed and will have a direct impact on BeB origins and energetics. Namely, if it is clearly established that $\mathrm{O} / \mathrm{Fe}$ is constant in Pop II, then the Be-O slopes point to a primary origin and no energetics problem is encountered, as has been well-established. However, the situation is clearly not settled, and moreover it is our purpose here to investigate the impact of the energy budget on the viability of standard GCR. With this motivation, and bearing in mind the observational caveats, we will use the $\mathrm{OH}$ data.

As seen in Fig. 2, the lowest metallicity point is $[\mathrm{O} / \mathrm{H}]=-1.74$, for which $\mathrm{Be} / \mathrm{H}=4.410^{-14}$. Using Fig. 1, the energy cost is $\dot{N}_{\mathrm{Be}} / \dot{W}=210^{-3}$ atom/erg. The data point has $\mathrm{Be} / \mathrm{O}=2.810^{-9}$ and using an oxygen yield per supernova of $\dot{M}(0) / \dot{\mathcal{N}}_{\mathrm{SN}}=2 M_{\odot}$ corresponding to $1.510^{56}$ atoms of oxygen, we find that we are required to produce $4.210^{47}$ atoms of Be per SN. We thus find that the cosmic ray energy input per supernova at this metallicity is $\epsilon=0.36$ foe/SN, corresponding to an efficiency of $\eta_{\mathrm{CR}}=0.36$ for $E_{\mathrm{SN}}=1$ foe. Thus we see that on the basis of Be production alone, one requires a total energy available per SN that is at about the level required to produce the observed flux of cosmic rays. In other words, we are able to produce the desired abundance of Be at low metallicity with an efficiency which is comparable that needed to accelerate cosmic rays.

The importance of the $N_{\mathrm{H}}$ factor in the energy per atom calculation is now clear, as it lowers the cosmic ray energy budget to within values that lie within the wide range allowed by present-day data and theory. Without this factor, the energy requirements inferred from Be would increase by about an order of magnitude. This would put the needed energy budget beyond the traditional estimates. It is worth noting, however, that even this case is not out of reach, if supernova blast energies are more typically 3 foe, and the cosmic ray efficiencies approach 50-60\%, notwithstanding the other uncertainties discussed in Sect. 2.2.

Let us compare the above result with the previous arguments made by Ramaty et al. (2000) and Parizot (2000). As mentioned in the introduction, Ramaty et al. base their argument on the evolution of $\mathrm{Be}$ with respect to Fe. At the low oxygen abundance of $[\mathrm{O} / \mathrm{H}]=-1.7$, we can assume a value of $[\mathrm{Fe} / \mathrm{H}]=-2.5$. Recall that the conclusion that secondary Be production was energetically excluded was based on the Fe yield, the constancy of $\mathrm{Be} / \mathrm{Fe}$, and the determination of the number of Be atoms per erg produced per supernova. At this metallicity, the $\mathrm{Be} / \mathrm{O}$ ratio of $2.810^{-9}$ corresponds to a ratio of $\mathrm{Be} / \mathrm{Fe}$ of $3.210^{-7}$ and is significantly below the constant mean value of $10^{-6}$. This is a result of the fact that the data show that the $\mathrm{Be} / \mathrm{Fe}$ is not a constant with respect to $\mathrm{Fe} / \mathrm{H}$, but decreases with decreasing metallicity (though slowly) and that there is considerable dispersion in the data. Had we used the estimate of energy requirement for secondary production of Ramaty et al. (2000) 
(4 $10^{-4}$ atoms/erg at this metallicity), we would have concluded that $\left(1.7 \times 2.8 \quad 10^{-9}\right)\left(\begin{array}{lll}1.5 & 10^{56}\end{array}\right) / 4 \quad 10^{-4}=$ $1.710^{51} \mathrm{ergs} / \mathrm{SN}$ is required. This may or may not be too much depending on the actual energy available. We have typically assumed that the total energy available in the explosion is $10^{51} \mathrm{erg}$, but this is uncertain by a factor of at least 3 , and if the available energy is actually 3 times higher, there is no energetics problem for the lowest metallicity points (and lowest Be) points observed. It is however very likely that primary sources are required at these low metallicities in order to explain the scatter in the data. Note that as argued above, the energy required per Be atom is significantly reduced when density effects are taken into account. In that case, energetics is unable to provide a strong indication as to the primary vs. secondary nature of Be production.

Parizot (2000) also provided an energetics argument against secondary production. We find several differences in the simple estimate which is used to preclude secondary production. First, at low metallicity, Parizot assumes a $\mathrm{Be} / \mathrm{O}$ ratio of $410^{-9}$, where it is in fact $2.810^{-9}$ (this stems in part from his incorrect value of the solar oxygen abundance). He further assumes that the maximum energy available for Be production is $10^{50} \mathrm{erg} / \mathrm{SN}$ corresponding to an efficiency of $10 \%$. This could be $3-5$ times higher and as noted above, the total SN energy could be 3 times higher. Finally his choice of a spectrum is far more pessimistic than even that used in Ramaty et al. and assumes a high energy cut off of $0.5 \mathrm{GeV}$. Thus from the argument given in Parizot (2000), one can not place a constraint on the secondary nature of Be.

For late times and high metallicities, the approximations of no Be astration and a constant Be-O slope break down. A more refined analysis is needed. Given the data, what is actually observable is the $\mathrm{Be} / \mathrm{O}$ ratio, which is in general not set by the ratio of mass change rates $\dot{N}(\mathrm{Be}) / \dot{N}(\mathrm{O})$, but rather $\mathrm{Be} / \mathrm{O}$. Since these observables connect not to rates but to integral quantities, they do not allow for direct measure of the instantaneous cosmic ray energy input $\dot{W}_{\mathrm{CR}}$. Instead, the data can determine the time-averaged value

$\langle\epsilon\rangle=\frac{W_{\mathrm{CR}}(t)}{\mathcal{N}_{\mathrm{SN}}(t)}=\frac{\int_{0}^{t} \mathrm{~d} t^{\prime} \dot{W}_{\mathrm{CR}}}{\int_{0}^{t} \mathrm{~d} t^{\prime} \dot{\mathcal{N}}_{\mathrm{SN}}}=\frac{\int_{0}^{t} \mathrm{~d} t^{\prime} \dot{\mathcal{N}}_{\mathrm{SN}} \epsilon}{\int_{0}^{t} \mathrm{~d} t^{\prime} \dot{\mathcal{N}}_{\mathrm{SN}}}$.

Within the model, we assume (and then test) a constant energy budget $\epsilon=\epsilon_{0}=$ const, and thus $\langle\epsilon\rangle=\epsilon_{0}$ as well. However, this quantity can also be calculated in a way that makes explicit use of the observed Be abundances.

We can calculate the (time-averaged) energetics as follows:

$\frac{W_{\mathrm{CR}}}{\mathcal{N}_{\mathrm{SN}}}=\left[\frac{W_{\mathrm{CR}}}{N(\mathrm{Be})}\right]_{\mathrm{th}}\left[\frac{N(\mathrm{Be})}{N(\mathrm{O})}\right]_{\mathrm{obs}}\left[\frac{N(\mathrm{O})}{\mathcal{N}_{\mathrm{SN}}}\right]_{\mathrm{th}}$.

The cosmic ray input energy per supernova is thus calculable given (1) this "energy per atom" ratio, (2) the observed $\mathrm{Be} / \mathrm{O}$ ratio, and (3) the oxygen output per supernova. In this way, one can use the observed Be trends versus $\mathrm{O}$ (or $\mathrm{Fe}$ ) to constrain the cosmic ray energy input per supernova over all epochs for which abundances are available. To do this, we rewrite Eq. (28), noting that in the code all quantities in Eq. (28) are computed explicitly. Thus we are free to rearrange terms and write

$$
\begin{aligned}
\frac{W_{\mathrm{CR}}}{\mathcal{N}_{\mathrm{SN}}} & =\left[\frac{N(\mathrm{Be})}{N(\mathrm{O})}\right]_{\mathrm{obs}}\left[\frac{N(\mathrm{O})}{N(\mathrm{Be})}\right]_{\mathrm{th}} \epsilon_{\mathrm{th}} \\
& =\frac{(\mathrm{Be} / \mathrm{O})_{\mathrm{obs}}}{(\mathrm{Be} / \mathrm{O})_{\mathrm{th}}} \epsilon_{\mathrm{th}}
\end{aligned}
$$

where $\epsilon_{\mathrm{th}}$ is the theoretical efficiency (and must be less than 30-50\%). Thus the true needed efficiency $\epsilon$ will exceed the theoretical efficiency only if the observed $\mathrm{Be} / \mathrm{O}$ ratios are greater than the theoretically predicted ones. That is if there is significant (upwards) scatter in the Be vs. O data, or there is a true excess in $\mathrm{Be} / \mathrm{H}$ at low metallicity, will we find that energetics preclude the secondary production of Be as was argued in FOVC.

Thus the energetics entirely reflects the agreement of the abundance trends. If the data are fit within the errors, then the right side of Eq. (30) is constant in metallicity, consistent with the assumption of constant $W_{\mathrm{CR}} / \mathcal{N}_{\mathrm{SN}}$. This is the case for primary models of, e.g., VangioniFlam et al. (1998) or Ramaty et al. (2000). This agreement with the data also holds case for standard GCR models with changing $\mathrm{O} / \mathrm{Fe}$, at least down to metallicities $[\mathrm{O} / \mathrm{H}] \simeq-1.75$. At lower metallicities, the data is not conclusive but seems to suggest the need for a primary component (FOVC). If this is borne out by improved data, then the need for a primary component of Be will also be reflected in a energetics problem for the standard GCR model at these metallicities. Careful observations at these metallicities are thus crucial and encouraged. In any case, the standard GCR model certainly provides an acceptable fit to the Be-O data above about $[\mathrm{O} / \mathrm{H}] \simeq-1.75$ (or $[\mathrm{Fe} / \mathrm{H}] \simeq-2.5$ ), and thus suffers no energetics problem in this regime.

To illustrate this conclusion, we can calculate the energetics for all of the data points illustrated in Fig. 2. We implement Eq. (30) for each data point, using our model to calculate $[\epsilon /(\mathrm{Be} / \mathrm{O})]_{\mathrm{th}}$, and the data for $(\mathrm{Be} / \mathrm{O})_{\mathrm{obs}}$. The result appears in Fig. 3, both with and without normalizing the Be solar abundance to its observed value. We see that in the standard case, there is scatter in the data but the mean remains close to the solar value, which is around 0.08 foe/SN.

Combining these factors, the solar beryllium and oxygen abundances give a present-day cosmic ray energy budget per supernova which spans a range of

$\epsilon=0.5 \pm 0.4$ foe $\quad($ per SN)

(using the weighted mean and the $2-\sigma$ weighted sample variance) or an efficiency of $\eta_{\mathrm{CR}}=(50 \pm 40) \%$ for a supernova blast wave energy of 1 foe. This number is consistent, within uncertainties, with our estimate of $\eta_{\mathrm{CR}}=16 \%$ using the observed cosmic ray flux (Eq. (14)). This concordance means that the present-day Be abundance is consistent with the energy that has gone into the cosmic rays up 


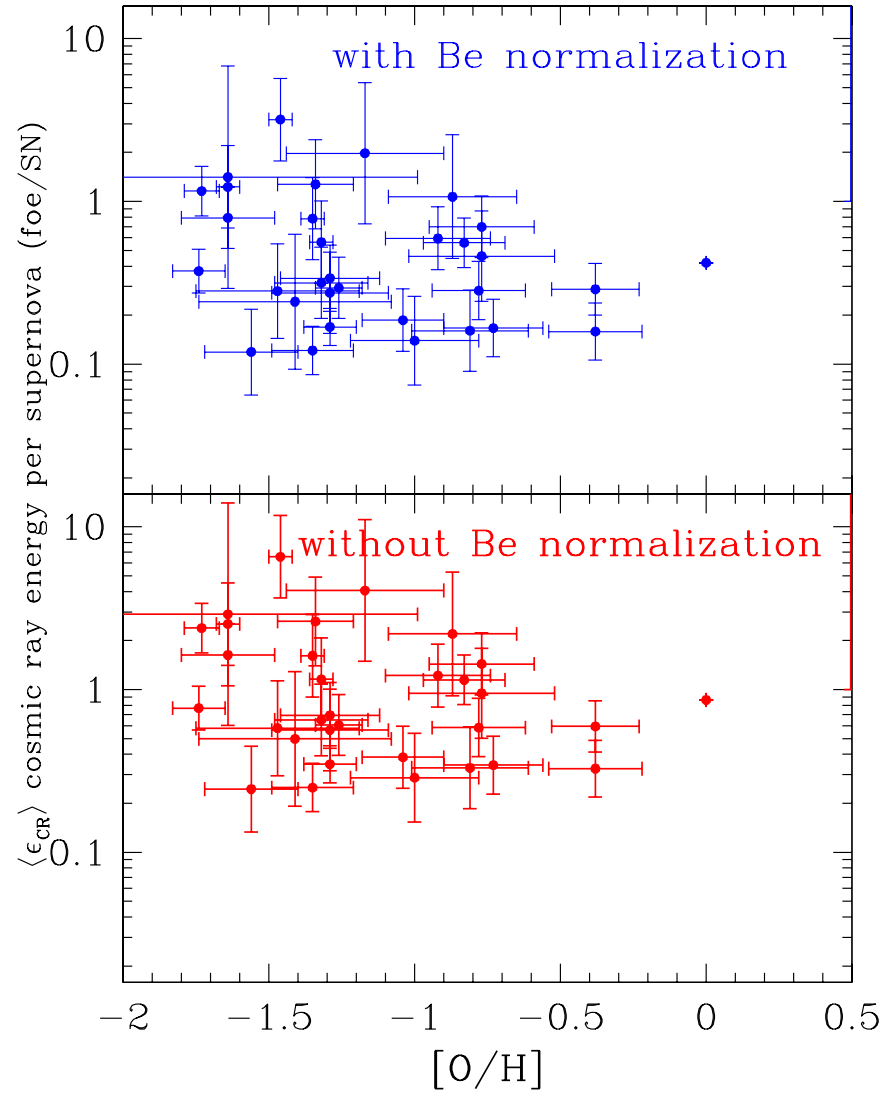

Fig. 3. Energetic requirements inferred point-by-point from the Be-O data and the model shown in Fig. 2. The upper panel is for the standard model which normalizes the Be point to solar; the lower panel does not use this scaling

to the present epoch. Thus, standard Galactic cosmic rays are able to account for both the present-day Be abundance as well as the associated energy, within the described uncertainties.

An important but somewhat technical detail concerns the normalization of the theory curves. The approach we have taken in this and previous work is to normalize the curves by demanding that the Be abundance at solar metallicity is equal to the solar Be abundance. Physically, this amounts to determining the scaling between the global average Galactic flux at the present epoch and the present flux at the solar system. The normalization factor is about 0.5 , i.e., the solar flux is slightly high. This factor has been included in Fig. 1. If we did not include this factor, we would both raise the entire $\mathrm{Be}-\mathrm{O}$ curve and increase the energy requirement by the same factor of $1 / 0.5=2$. The overall mean is thus higher in the non-normalized case, at $1.0 \pm 0.8$ foe/SN. This is just at the fiducial (but poorly determined) supernova mechanical energy budget, but of course the lack of normalization also leads to a poorer fit to the Be-O data. Thus we see an illustration of the connection between energetics and the Be-O fit: a poorer agreement with Be-O data goes hand in hand with poorer agreement with energetics requirements.

Finally, we recall that the energy budget we have determined depends sensitively on the cosmic ray spectrum adopted. We have used a $p^{-2.2}$ source, which we consider to be a fair representation of the experimental determinations; however, the present cosmic ray data allow for other spectral forms and different energetic requirements. For example, used by Ramaty et al. (2000) is more energetically efficient by a factor of 2.5 (i.e., $\langle E q\rangle$ is $40 \%$ of the value using our adopted spectrum), as noted in Sect. 2.2. Thus, using that spectrum, the energy requirements would drop by a factor of 2.5 , both for the present cosmic rays and for the past as derived form Be. Thus, while the ratios of past/present values would be similar, but the absolute energy requirements would be reduced. Using the Parizot spectrum (with $500 \mathrm{MeV}$ cutoff) would reduce this requirement yet further.

To summarize, for models with supernova-powered cosmic rays, the fit of the energetics is completely determined by, and follows from, the fit of the observed Be-O data. Models which fit the data (and have $\psi \propto \dot{\mathcal{N}}_{\mathrm{SN}}$ ) do not have an energetics problem in the past if they do not have one now. Conversely, models which do not fit the data (in particular, those which underpredict the Be abundance) can also be expected to have energetic difficulties over the Be-O regimes which are poorly fit.

\section{Lithium-6 constraints on energetics}

We can repeat this analysis for other cosmic-ray produced elements. For ${ }^{7} \mathrm{Li}$ and ${ }^{11} \mathrm{~B}$, the results are complicated by the fact that these elements are produced in other sources. However, ${ }^{6} \mathrm{Li}$, like $\mathrm{Be}$, is a pure cosmicray element, and thus the energetics evaluation should proceed as it does for Be. We first consider the presentday energetics (the analog of Eq. (31)). For ${ }^{6} \mathrm{Li}$, we have $\dot{Q}\left({ }^{6} \mathrm{Li}\right) / \dot{W}_{\mathrm{CR}}=0.05$ atom/erg using the solar value from Fig. 1 , and $\left({ }^{6} \mathrm{Li} / \mathrm{O}\right)_{\odot}=1.810^{-7}$. Finally, we again take an average of $2 M_{\odot}$ of oxygen ejected per supernova, or $1.510^{56}$ atoms. Together, this gives

$\epsilon=0.54$ foe $\quad($ per SN $)$.

This is comparable to, albeit somewhat higher, than the other estimates we have made. Also, as with the Be analysis, using a source spectrum such as that of Ramaty et al. (2000) would lower this requirement by a factor of 2.5.

Regarding the energetics of ${ }^{6} \mathrm{Li}$ in the past, the analysis of the previous section carries over: the efficiency remains constant if the theory and data agree. The data for ${ }^{6} \mathrm{Li}$ in Pop II stars are difficult to obtain, and thus this is a less definitive test. However, as shown by Fields \& Olive (1999b), the predictions of standard GCR agree with the presently available data. Thus we see that the ${ }^{6} \mathrm{Li}$ constraints on standard GCR are quantitatively similar to those of $\mathrm{Be}$, and demonstrate the basic consistency of the analysis.

Note that ${ }^{6} \mathrm{Li}$ can be made via the $\alpha+\alpha$ fusion reaction. This production mode is always primary since most helium in the ISM is primordial and hence predates the Galaxy. ${ }^{6} \mathrm{Li}$ is thus primary in both the standard GCR scenario of particle acceleration from the ISM, as well as 
in the scenario of GCR origin dominated by superbubbles (Ramaty et al. 2000). An approach which synthesizes aspects of these two outlooks adds to the standard GCR scenario a component of energetic particles (EPs) which could also originate from superbubbles (for a review see Vangioni-Flam et al. 2000). The EP component is highly enriched in $\alpha$ particles since its origin is in the massive star ejecta which fill the superbubbles. Due to this enhancement, the EP component can explain the ${ }^{6} \mathrm{Li}$ data with an even lower energy efficiency than that required above for GCR particles. Further ${ }^{6} \mathrm{Li}$ data would help distinguish between the EP and GCR scenarios for the $\alpha$-component of early Galactic cosmic rays.

\section{Conclusions}

In this paper, we have reviewed the past and present energy budget for standard Galactic cosmic rays We determine present-day cosmic ray power requirements from the observed cosmic ray flux and confinement. Comparing this to the fiducial $10^{51} \mathrm{erg}$ of kinetic energy in a supernova blast, we find the efficiency of cosmic ray acceleration is about $30 \%$. This value cannot be calculated to a precision better than a factor of about 3 , given the uncertainties in the cosmic ray and Galactic input parameters. Nevertheless, we find that this value is consistent with estimates derived from present (solar) ${ }^{9} \mathrm{Be}$ and ${ }^{6} \mathrm{Li}$ values.

We have extended our analysis to the study the cosmic ray acceleration efficiency in the early Galaxy, as encoded in the Be abundances of metal-poor stars. We find that energy considerations are indeed powerful, and allow us to derive the usual $\phi \propto \psi$ scaling. We also find that in general, the requirement that models satisfy energetic requirements is equivalent to the requirement that the models produce $\mathrm{LiBeB}$ evolution which fits the abundances data, if SN are the cosmic ray acceleration engines.

Furthermore, constructing a careful and consistent model for the energetics of cosmic ray acceleration and LiBeB production has an additional benefit: it brings to the surface key underlying physical issues and assumptions regarding early Galactic LiBeB production. Specifically, we have emphasized that the ISM gas content, and thus the total number of ISM atoms, was higher in the early Galaxy. Thus, for a fixed metallicity, the total Galactic Be production is enhanced due to the increased total number of targets. This effect has been neglected up to now but can play a crucial role in the energetics: due to this effect, the number of Be atoms produced per cosmic ray erg should be multiplied by about a factor 10 in the early Galaxy compared to the estimates lacking this effect. Consequently, the energy test is less stringent than previously thought. Note that one could invoke still more complications in the form of early Galactic evolution of cosmic ray confinement properties, or a lack of equilibrium between cosmic ray sources and sinks; further study of these issues is best done in a consistent model of cosmic ray acceleration and propagation in a Galaxy which evolves in time and space. In the absence of such a model, the current "leaky box" picture that has been adopted for Be studies, with constant cosmic ray confinement parameters, leads to the factor of $\sim 10$ increase in Be production efficiency we have described. With this effect, the standard GCR model is able both to fit the Be-Fe data, and to maintain a reasonable (and roughly constant) acceleration efficiency over the history of the Galaxy at least down to $[\mathrm{Fe} / \mathrm{H}] \simeq-2.5$.

Thus we find that energetic arguments are extremely useful tools in analyzing $\mathrm{LiBeB}$ production in the early Galaxy, and thereby to examine questions of cosmic ray origin. However, contrary to some analyses, we find that the standard GCR scenario does not suffer energetics problems, and thus that energetics alone cannot rule this model out. Therefore, the key of the origin of early Galactic cosmic rays and $\mathrm{LiBeB}$ (i.e., a primary or secondary process?) remains in the hands of the observers. Specifically, it is crucial to reconcile the different measurements of oxygen (and thus BeB-O and $\mathrm{O}-\mathrm{Fe}$ ) in halo stars. The determination of reliable oxygen abundances is to be ranked as a first priority of the abundance observation programs.

Indeed, looking towards the future, we can hope to turn the problem around. With reliable and accurate oxygen data, we can establish the origin of $\mathrm{LiBeB}$ and cosmic rays in the early Galaxy. With this knowledge in hand, we can then use the detailed LiBeB abundance patterns and scatter to learn more about the physical conditions of gas and accelerated particles in the early Galaxy.

Acknowledgements. We thank Tom Jones and Vasiliki Pavlidou for fruitful discussions. This work was supported in part by DoE grant DE-FG02-94ER-40823 at the University of Minnesota.

\section{References}

Asplund, M., 24th meeting of the IAU, Joint Discussion 8, August 2000, Manchester, UK, 8, E8 [astro-ph/0011043]

Berezhko, E. G., \& Völk, H. J. 1997, Astropart. Phys., 7, 183

Boesgaard, A. M., Deliyannis, C. P., King, J. R., et al. 1999, AJ, 117, 1549

Boesgaard, A. M., King, J. R., Deliyannis, C. P., \& Vogt, S. S. 1999, AJ, 117, 492

Boesgaard, A., \& King, J. R. 1993, AJ, 106, 2309

Brerezinskǐ̌, V. S., Bulanov, S. V., Dogiel, V. A., Ginzburg, V. L., \& Ptuskin, V. S. 1990, Astrophysics of Cosmic Rays (Amsterdam: North-Holland)

Buckley, J., Dwyer, J., Mueller, D., Swordy, S., \& Tang, K. K. 1994, ApJ, 429, 736

Carretta, E., Gratton, R. G., \& Sneden, C. 2000, A\&A, 356, 238

Cassé, M., Lehoucq, R., \& Vangioni-Flam, E. 1995, Nature, 373,38

Connell, J. J. 1998, ApJL, 501, L59

Combi, J. A., Romero, G. E., \& Benaglia, P. 1998, A\&A, 333, L91

Dragicevich, P. M., Blair, D. G., \& Burman, R. R. 1999, MNRAS, 302, 693

Drury, L. O., Markiewicz, W. J., \& Voelk, H. J. 1989, A\&A, 225, 179 
Engelmann, J. J., Ferrando, P., Soutoul, A., Goret, P., \& Juliusson, E. 1990, A\&A, 233, 96

Fields, B. D., \& Olive, K. A. 1999a, ApJ, 516, 797

Fields, B. D., \& Olive, K. A. 1999b, New Astron., 4, 255

Fields, B. D., Olive, K. A., Vangioni-Flam, E., \& Cassé, M. 2000, ApJ, 540, 930 (FOVC)

Fulbright, J. P., \& Kraft, R. P. 1999, AJ, 118, 527

Garcia-Muñoz, M., Simpson, J. A., Guzik, T. G., Wefel, J. P., \& Margolis, S. H. 1987, ApJS, 64, 269

Gilmore, G., Gustafsson, B., Edvardsson, B., \& Nissen, P. E. 1992, Nature, 357, 379

Gratton, R. G., Carretta, E., Matteucci, F., \& Sneden, C. 2000, A\&A, 358, 671

Henderson, A. P., Jackson, P. D., \& Kerr, F. J. 1982, ApJ, 263, 116

Higdon, B., Lingenfelter, R. E., \& Ramaty, R. 1998, ApJ, 509, L33

Hobbs, L. M., \& Thorburn, J. A. 1996, AJ, 111, 2106

Israelian, G., García-López, R. J., \& Rebolo, R. 1998, ApJ, 507,805

Israelian, G., et al. 2001, ApJ, in press [astro-ph/0101032]

Janni, J. F. 1982, Atomic Data Nucl. Data Tables, 27, 34

King, J. R. 2000, AJ, 120, 1056

Koyama, K., et al. 1995, Nature, 378, 255

Lukasiak, A., Ferrando, P., McDonald, F. B., \& Webber, W. R. 1994, ApJ, 423, 426

Northcliffe, L. C., \& Schilling, R. F. 1970, Nucl. Data Tables, A7, 233

Mishenina, T. V., Korotin, S. A., Klochkova, V. G., \& Panchuk, V. E. 2000, A\&A, 353, 978

Molaro, P., Bonifacio, P., Castelli, F., \& Pasquini, L. 1997, A\&A, 319, 593

Mori, M. 1997, ApJ, 4478, 225

Parizot, E. 2000, A\&A, in press [astro-ph/0006099]

Primas, F. 1995, Ph.D. Thesis

Primas, F., Molaro, P., Bonifacio, P., \& Hill, V. 2000, A\&A, 362,666

Primas, F., Asplund, M., Nissen, P. E., \& Hill, V. 2000, A\&AL, in press [astro-ph/0009482]

Ramaty, R., Kozlovsky, B., Lingenfelter, R. E., \& Reeves, H. 1997, ApJ, 488, 730

Ramaty, R., Kozlovsky, B., \& Lingenfelter, R. E. 1998, Phys. Today, 51, 4, 30

Ramaty, R., \& Lingenfelter, R. E. 1999, in LiBeB Cosmic Rays and Related X- and Gamma-Rays, ed. Ramaty et al., ASP, 171, 104

Ramaty, R., Scully, S. T., Lingenfelter, R. E., \& Kozlovsky, B. 2000, ApJ, 534, 747

Rebolo, R., et al. 1993, in Origin and Evolution of the Elements, ed. N. Prantzos, E. Vangioni-Flam, \& M. Cassé (Cambridge University Press), 149

Rebolo, R., Molaro, P., Abia, C., \& Beckman J. E. 1988, A\&A, 193, 193

Reeves, H., Fowler, W. A., \& Hoyle, F. 1970, Nature

Ryan, S., Bessell, M., Sutherland, R., \& Norris, J. 1990, ApJ, 348, L57

Ryan, S. G., Norris, J. E., Bessell, M. S., \& Deliyannis, C. 1992, ApJ, 388, 184

Ryter, C., Reeves, H., Gradsztajn, E., \& Audouze, J. 1970, A\&A, 8, 389

Scalo, J. M. 1986, Fund. Cosmic Phys., 11, 1

Simpson, J. A., \& Garcia-Munoz, M. 1988, Space Sci. Rev., 46, 205

Takeda, Y., Takada-Hidai, M., Sato, S., et al. 2000, ApJ, submitted [astro-ph/0007007]

Tanimori, T., et al. 1998, ApJ, 497, L25

Timmes, F. X., Diehl, R., \& Hartmann, D. H. 1997, ApJ, 479, 760

Vangioni-Flam, E., Cassé, M., \& Audouze, J. 2000, Phys. Rep., 333-334, 365

Vangioni-Flam, E., Cassé, M., Fields, B. D., \& Olive, K. A. 1996, ApJ, 468, 199

Vangioni-Flam, E., Ramaty, R., Olive, K. A., \& Cassé, M. 1998, AA, 337, 714

Webber, W. R. 1998 ApJ, 506, 329 